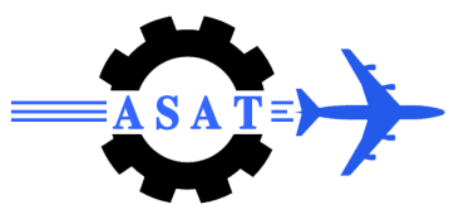

\title{
Optimum Nonlinear Response of Composite Panels Subjected to Aerodynamic and Thermal Loadings
}

\author{
S. Saleh*, A-N. Zayed ${ }^{\dagger}$, H.H. Ibrahim $*$ W.A. Wahba ${ }^{\S}$
}

\begin{abstract}
A nonlinear finite element model is provided for the thermal post buckling and linear flutter behavior of composite panels. Panel subjects to combined aerodynamic and thermal loads. The governing equations are derived using the classical plate theory and the principle of virtual work. The effect of large deflection is included in the formulation through the von Kármán nonlinear strain-displacement relations. To account for the temperature dependence on material properties, the thermal strain is stated as an integral quantity of the thermal expansion coefficient with respect to temperature. The aerodynamic pressure is modeled using the quasi-steady first order piston theory. The Newton-Raphson iteration method is employed to obtain the nonlinear aero-thermal post-buckling deflections, and a frequency-domain solution is presented to predict the critical dynamic pressure at different elevated temperatures. Finally, numerical results are provided to depict the optimum lamination scheme in order to maximize the aero-thermal stability of such panels. The optimum solution is obtained by Genetic Algorithms.
\end{abstract}

Keywords: Thermal buckling, Supersonic panel flutter, Nonlinear FEM, Genetic Algorithms.

\section{Introduction}

The external skins of supersonic flight vehicles experience high temperature rise due to aerodynamic heating. A flat plate panel with symmetric 8-plies composite material is studied. This panel induces thermal buckling and panel flutter that occur at statically and dynamically instability, respectively.

Instability due to thermal expansion reshapes the skin of panel which reduces the aerodynamic performance. The fundamental frequency which has dependency in dynamically instability is studied. Finally, genetic algorithm is the distinct optimization algorithm improves the aero-thermal stability and delaying the critical buckling temperature.

Ibrahim et. al. [1] studied the thermal buckling and free vibration behavior of shape memory alloy (SMA) with hybrid composite panel. SMA improved the stiffness of plate, the critical temperature was increased and the thermal large deflection was decreased. Lee et. al. [2] studied the design of a thick laminated composite plate subjected to a thermal buckling load under a uniform temperature distribution. A one-dimensional search method was used to find optimal fiber orientation and optimal thickness, this method is a golden section method. Kan and Kim [3] investigated the minimum-weight design of compressively loaded composite

\footnotetext{
*Egyptian Armed Forces, Egypt. shmtcg40@ hotmail.com

† Professor, Modern Academy in Maadi, Egypt.

Assistant Professor, Aeronautical Department, Emirates Aviation College, Dubai, UAE.

$\S$ Egyptian Armed Forces, Egypt.
} 
plates under constrained post-buckling strength. Paluch et al. [4] presented a procedure allowing layout and thickness optimization of composite structures by means of a genetic algorithm and a finite-element program. Spallino and Thierauf [5] studied the requirements of aerospace structure components that were able to withstand the external environmental loads without loss of stability. Faria and Almedia [6] analyzed the problem of optimizing buckling loads of plates with variable thickness within the scope of a novel approach where the variability or uncertainty of the loading distribution was taken into consideration. Lanhe [7] derived equilibrium and stability equations of moderately thick simply supported rectangular plate made of Functionally Graded Material (FGM) under thermal loads based on the first order shear deformation theory. Mukherjee et al. [8] studied panels of re-entry vehicles subjected to a wide range of flow conditions during ascent and re-entry phases. The flow could vary from subsonic continuum flow to hypersonic rare-field flow with wide ranging of dynamic pressure and aerodynamic heating. Ibrahim et al. [9] presented a finite element solution for the thermal buckling and nonlinear flutter performance of thin functionally graded material panels under combined aerodynamic and thermal loads. At certain temperature, the decreasing of volume fraction exponent enhanced flutter characteristics through increasing the critical dynamic pressure.

\section{Finite Element Formulation of Thermal Postbuckling of Composite Material Panels}

The equations of motion with the consideration of moderately large deflection and temperature-dependent material properties are derived for a composite plate subject to aerodynamic and thermal loadings. To account for the temperature dependence on material properties, a cumulative thermal strain is adopted for the calculation of the thermal deflection and stresses in the plate. The element used in this study is the rectangular, four-node, $C^{0}$, nonconforming element (for the bending DOF)

The nodal degrees of freedom vector of a four-noded rectangular plate element can be written as

$$
\left\{w, \frac{\partial w}{\partial x}, \frac{\partial w}{\partial y}, \frac{\partial^{2} w}{\partial x \partial y}, u, v\right\}^{T}=\left\{\begin{array}{l}
\left\{w_{b}\right\} \\
\left\{w_{m}\right\}
\end{array}\right\}
$$

where $\mathrm{w}$ is the transverse displacement, $\mathrm{u}$ and $\mathrm{v}$ are the membrane displacements in the $\mathrm{x}$ and $\mathrm{y}$ directions respectively, $\left\{\mathrm{w}_{\mathrm{b}}\right\}$ is the transverse displacement vector, and $\left\{\mathrm{w}_{\mathrm{m}}\right\}$ is the membrane displacement vector

The displacement/nodal-displacement relation can be presented in terms of interpolation function matrices $\left[N_{w}\right],\left[N_{u}\right]$, and $\left[N_{v}\right]$.

$w=\left[N_{w}\right]\left\{w_{b}\right\}, u=\left[N_{u}\right]\left\{w_{m}\right\}, v=\left[N_{v}\right]\left\{w_{m}\right\}$

Nonlinear Strain-Displacement Relation the in-plane strains and curvatures, based on the von Kármán moderately large deflection and classical plate theory, are given by Timoshenko and Woinowsky-Krieger [10] and Ventsel and Krauthammer [11] [11] 


$$
\left\{\begin{array}{c}
\varepsilon_{x} \\
\varepsilon_{y} \\
\gamma_{x y}
\end{array}\right\}=\left\{\begin{array}{c}
\frac{\partial u}{\partial x} \\
\frac{\partial v}{\partial y} \\
\frac{\partial u}{\partial y}+\frac{\partial v}{\partial x}
\end{array}\right\}+\left\{\begin{array}{c}
\frac{1}{2}\left(\frac{\partial w}{\partial x}\right)^{2} \\
\frac{1}{2}\left(\frac{\partial w}{\partial y}\right)^{2} \\
\frac{\partial w}{\partial x} \frac{\partial w}{\partial y}
\end{array}\right\}+z\left\{\begin{array}{c}
-\frac{\partial^{2} w}{\partial x^{2}} \\
-\frac{\partial^{2} w}{\partial y^{2}} \\
-2 \frac{\partial^{2} w}{\partial x \partial y}
\end{array}\right\}
$$

or, in compact form,

$$
\{\varepsilon\}=\left\{\varepsilon_{m}\right\}+\left\{\varepsilon_{\theta}\right\}+z\{\kappa\}
$$

where $\varepsilon_{m}, \varepsilon_{\theta}$, and $\kappa$ are the membrane linear strain vector, the membrane nonlinear strain vector due to large deflection, and the bending strain vector, respectively.

Stress-Strains Relation for a general $\mathrm{k}^{\text {th }}$ lamina with an orientation angle $\Phi \square$ become

$$
\left.\{\sigma\}^{\mathrm{k}}=\left\{\begin{array}{c}
\sigma_{x} \\
\sigma_{y} \\
\tau_{x y}
\end{array}\right\}=[\bar{Q}(T)]^{k}\left(\left\{\begin{array}{c}
\varepsilon_{x} \\
\varepsilon_{y} \\
\gamma_{x y}
\end{array}\right\}-\int_{\text {Tref }}^{T}\left\{\begin{array}{c}
\alpha_{x}(\tau) \\
\alpha_{y}(\tau) \\
\alpha_{x y}(\tau)
\end{array}\right\}^{k}\right) d \tau\right)
$$

and

$$
[Q(T)]=\left[\begin{array}{ccc}
Q_{11}(T) & Q_{12}(T) & 0 \\
Q_{21}(T) & Q_{22}(T) & 0 \\
0 & 0 & Q_{66}(T)
\end{array}\right]
$$

where

$$
[\bar{Q}]=\left[T_{\sigma}(\phi)\right]^{-1}[Q]\left[T_{\varepsilon}(\phi)\right], \quad\left\{\begin{array}{c}
\alpha_{\mathrm{x}} \\
\alpha_{\mathrm{y}} \\
\alpha_{\mathrm{xy}}
\end{array}\right\}=\left[\mathrm{T}_{\varepsilon}(\varphi)\right]^{-1}\left\{\begin{array}{c}
\alpha_{1} \\
\alpha_{2} \\
0
\end{array}\right\}
$$

$\left[T_{\sigma}(\phi)\right]=\left[\begin{array}{ccc}c^{2} & s^{2} & 2 s c \\ s^{2} & c^{2} & -2 s c \\ -s c & s c & c^{2}-s^{2}\end{array}\right]$, and $\quad\left[T_{\varepsilon}(\phi)\right]=\left[\begin{array}{ccc}c^{2} & s^{2} & s c \\ s^{2} & c^{2} & -s c \\ -2 s c & 2 s c & c^{2}-s^{2}\end{array}\right]$

The resultant force and moment vectors per unit length are defined as, [12]

$$
(\{N\},\{M\})=\int_{-h / 2}^{h / 2}\{\sigma\}^{k}(1, z) d z
$$

or

$$
\left\{\begin{array}{l}
N \\
M
\end{array}\right\}=\left[\begin{array}{ll}
A & B \\
B & D
\end{array}\right]\left\{\begin{array}{c}
\varepsilon^{o} \\
\kappa
\end{array}\right\}-\left\{\begin{array}{l}
N_{T} \\
M_{T}
\end{array}\right\}
$$


where $\mathrm{h}$ is the plate thickness, and $[\mathrm{A}],[\mathrm{B}]$, and $[\mathrm{D}]$ are the laminate stiffness matrices. They are defined as

$$
([A],[B],[D])=\sum_{k=1}^{n} \int_{Z_{k+1}}^{Z_{k}}[\bar{Q}(T)]^{k}\left(1, z, z^{2}\right) d z
$$

The vectors $\left\{\mathrm{N}_{\mathrm{T}}\right\}$, and $\left\{\mathrm{M}_{\mathrm{T}}\right\}$ are the resultant force and moment vectors per unit length, which are due to the thermal stress. They can be expressed as, Reddy [13]

$$
\left.\left(\left\{N_{T}\right\},\left\{M_{T}\right\}\right)=\sum_{k=1}^{n} \int_{Z_{k+1}}^{Z_{k}}[\bar{Q}]\right]^{k}\left(\int_{\operatorname{Tref}}^{T}\left\{\begin{array}{c}
\alpha_{x}(\tau) \\
\alpha_{y}(\tau) \\
0
\end{array}\right\}^{k} d \tau\right)(1, z) d z,
$$

\section{Aerodynamic Loading}

Quasi-steady first-order piston aerodynamic theory is a verified aerodynamic model, by Ibrahim [14] and Dowell [15]

$$
P_{a}=-\left[\lambda \frac{D_{110}}{L^{3}} \frac{\partial w}{\partial x}+\frac{g_{a}}{\omega_{o}} \frac{D_{110}}{L^{4}} \frac{\partial w}{\partial t}\right]
$$

where

$$
\lambda=\frac{2 \mathrm{qL}^{3}}{\beta \mathrm{D}_{110}}, \mathrm{~g}_{\mathrm{a}}=\frac{\rho_{\mathrm{a}} \mathrm{V}\left(\mathrm{M}_{\infty}^{2}-2\right)}{\beta^{3} \rho h \omega_{\mathrm{o}}}=\sqrt{\lambda \mathrm{C}_{\mathrm{a}}}, \quad \omega_{\mathrm{o}}=\sqrt{\frac{\mathrm{D}_{110}}{\rho \mathrm{LL}^{4}}}, \mathrm{C}_{\mathrm{a}}=\left(\frac{\mathrm{M}_{\infty}^{2}-2}{\mathrm{M}_{\infty}^{2}-1}\right)^{2} \frac{\mu}{\beta}
$$

where $\mathrm{P}_{\mathrm{a}}$ is the aerodynamic pressure, $\mathrm{M}_{\infty}$ is the undisturbed Mach number, $q=\frac{1}{2} \rho_{a} V^{2}$ is the dynamic pressure, $\rho_{\alpha}$ is the air mass density, $\beta=\sqrt{M_{\infty}^{2}-1}$, and $\mathrm{w}$ is the panel transverse displacement, $\mathrm{D}_{110}$ is the first entry in the laminate bending stiffness matrix $\mathrm{D}(1,1), \mathrm{L}$ is the panel length in the flow direction; and $\lambda$ is the non-dimensional dynamic pressure.

\section{Governing Equation}

Governing equation states that the work done by internal and external forces (including inertia forces) through an infinitesimal virtual displacement is zero, Ibrahim [14]

$$
\delta W=\delta W_{\text {int }}-\delta W_{\text {ext }}=0
$$

The virtual work done by internal forces on a plate element is given by

$$
\begin{aligned}
& \delta W_{\mathrm{int}}=\int_{A}\left(\left\{\delta \varepsilon^{o}\right\}^{T}\{N\}+\{\delta \kappa\}^{T}\{M\}\right) d A \\
& \delta W_{\mathrm{int}}=\{w\}^{T}\left([K]-\left[K_{T}\right]+\frac{1}{2}[N 1]+\frac{1}{3}[N 2]\right)\{w\}-\{w\}^{T}\left\{P_{T}\right\}
\end{aligned}
$$

where $\{\mathrm{w}\}=\left\{w, \frac{\partial w}{\partial x}, \frac{\partial w}{\partial y}, \frac{\partial^{2} w}{\partial x \partial y}, u, v\right\}$ is the nodal-displacement vector; $[\mathrm{K}]$ and $\left[\mathrm{K}_{\mathrm{T}}\right]$ are the linear stiffness matrix and the thermal geometric stiffness matrix respectively; $\left[\mathrm{N}_{1}\right]$ and $\left[\mathrm{N}_{2}\right]$ are the first- and second-order nonlinear stiffness matrices, respectively; and $\left\{\mathrm{P}_{\mathrm{T}}\right\}$ is the thermal load vector. 
The virtual work done by external forces on a plate element, considering inertia, and aerodynamic, is

$$
\begin{aligned}
& \delta W_{e x t}=\int_{A}\left(\delta w_{b}\left(-\rho h \ddot{w}_{b}+P_{a}\right)+\delta u(-\rho h \ddot{u})+\delta v(-\rho h \ddot{v})\right) d A \\
& \delta W_{e x t}=-\{\delta w\}^{T}[M]\{w\}^{\bullet}-\{\delta w\}^{T}[G]\{w\}^{\bullet}-\{\delta w\}^{T} \lambda\left[A_{a}\right]\{w\}
\end{aligned}
$$

where $\mathrm{h}$ denotes the plate thickness, $[\mathrm{M}]$ is the mass matrix, $[\mathrm{G}]$ is the aerodynamic damping matrix, and $\left[\mathrm{A}_{\mathrm{a}}\right]$ is the aerodynamic influence matrix.

Substituting by equations (17), (19) into equation (15), yields

$$
\begin{aligned}
& {\left[\begin{array}{cc}
M_{b} & 0 \\
0 & M_{\mathrm{m}}
\end{array}\right]\left\{\begin{array}{l}
\ddot{W}_{b} \\
\ddot{W}_{m}
\end{array}\right\}+\left[\begin{array}{cc}
G & 0 \\
0 & 0
\end{array}\right]\left\{\begin{array}{l}
\dot{W}_{b} \\
\dot{W}_{m}
\end{array}\right\}+\left(\begin{array}{c}
\lambda\left[\begin{array}{cc}
A_{a} & 0 \\
0 & 0
\end{array}\right]+\left[\begin{array}{cc}
K_{b} & K_{\mathrm{bm}} \\
K_{\mathrm{mb}} & K_{\mathrm{m}}
\end{array}\right]-\left[\begin{array}{cc}
K_{T b} & 0 \\
0 & 0
\end{array}\right]+ \\
\frac{1}{2}\left[\begin{array}{cc}
N_{1 b} & N_{1 \mathrm{bm}} \\
N_{1 \mathrm{mb}} & 0
\end{array}\right]+\frac{1}{3}\left[\begin{array}{cc}
N_{2 b} & 0 \\
0 & 0
\end{array}\right]
\end{array}\right\}\left\{\begin{array}{l}
W_{b} \\
W_{m}
\end{array}\right\}} \\
& =\left\{\begin{array}{l}
P_{\mathrm{bT}} \\
P_{\mathrm{mT}}
\end{array}\right\}
\end{aligned}
$$

or simply

$$
[\mathrm{M}]\{\ddot{\mathrm{W}}\}+[\mathrm{G}]\{\dot{\mathrm{W}}\}+\left(\lambda\left[\mathrm{A}_{\mathrm{a}}\right]+[\mathrm{K}]-\left[\mathrm{K}_{\mathrm{T}}\right]+\frac{1}{2}[\mathrm{~N} 1]+\frac{1}{3}[\mathrm{~N} 2]\right)\{\mathrm{W}\}=\left\{\mathrm{P}_{\mathrm{T}}\right\}
$$

\section{Procedure of solution}

The solution of the governing equation (21) is assumed to be as follows

$$
\{W\}=\{W\}_{s}+\{W\}_{t}
$$

The homogeneous solution refers to a self-excited dynamic oscillation $\{W\}_{t}$, and the particular solution refers to the aero-thermal static equilibrium deflection $\{W\}_{s}$. Both deflections $\{W\}_{s}$ and $\{W\}_{t}$ are considered to be large.

Substituting by equation (22) into equation (21) results in:

$$
[\mathrm{M}]\left(\{\ddot{\mathrm{W}}\}_{\mathrm{s}}+\{\ddot{\mathrm{W}}\}_{\mathrm{t}}\right)+[\mathrm{G}]\left(\{\dot{\mathrm{W}}\}_{\mathrm{s}}+\{\dot{\mathrm{W}}\}_{\mathrm{t}}\right)+\left(\begin{array}{c}
\lambda\left[\mathrm{A}_{\mathrm{a}}\right]+[\mathrm{K}]-\left[\mathrm{K}_{\mathrm{T}}\right] \\
+\frac{1}{2}[\mathrm{~N} 1]_{\mathrm{s}+\mathrm{t}}+\frac{1}{3}[\mathrm{~N} 2]_{\mathrm{s}+\mathrm{t}}
\end{array}\right)\left(\{\mathrm{W}\}_{\mathrm{s}}+\{\mathrm{W}\}_{\mathrm{t}}\right)=\left\{\mathrm{P}_{\mathrm{T}}\right\}
$$

Resolve equation (23) to obtain it in the form of

$$
\begin{aligned}
& [M]\}(\ddot{W}\}_{t}+[G]\{\dot{W}\}_{t}+\left(\lambda\left[A_{a}\right]+[K]-\left[K_{T}\right]_{t}\right)\left(\{W\}_{s}+\{W\}_{t}\right)+\frac{1}{2}\left([N 1]_{s}+[N 1]_{t}\right)\left(\{W\}_{s}+\{W\}_{t}\right) \\
& \frac{1}{3}\left([N 2]_{s}+[N 2]_{t}+2[N 2]_{s t}\right)\left(\{W\}_{s}+\{W\}_{t}\right)=\left\{P_{T}\right\}
\end{aligned}
$$

Equation (24) represents the general equation for the thermal buckling and nonlinear flutter of a composite material plate under the combined effect of aerodynamic and thermal loads. The subscripts $\mathrm{s}$ and $\mathrm{t}$ indicate that the relevant matrix depends on the static or dynamic displacements, respectively. Separating the static and dynamic terms of Eq. (24), the following two equations can be obtained 


$$
\begin{gathered}
\left(\lambda\left[A_{a}\right]+[K]-\left[K_{T}\right]+\frac{1}{2}[N 1]_{s}+\frac{1}{3}[N 2]_{s}\right)\{W\}_{s}=\left\{P_{T}\right\} \\
{[M]\{\ddot{W}\}_{t}+[G]\{\dot{W}\}_{t}+\left(\lambda\left[A_{a}\right]+[K]-\left[K_{T}\right]+\frac{1}{2}[N 1]_{t}+\frac{1}{3}[N 2]_{t}\right)\{W\}_{t}} \\
+\left([N 1]_{s}+[N 2]_{s t}+[N 2]_{s}\right)\{W\}_{t}=0
\end{gathered}
$$

\section{Static Aero-Thermal Buckling}

In the following, the solution procedure using the Newton-Raphson method for the aerothermal post-buckling analysis of a composite material plate is presented.

Introducing the function $\Psi(W)$ to equation (25),

$$
\Psi(W)=\left(\lambda\left[A_{a}\right]+[K]-\left[K_{T}\right]+\frac{1}{2}[N 1]+\frac{1}{3}[N 2]\right)\{W\}-\left\{P_{T}\right\}=0
$$

Equation (27) is expressed as a Taylor expansion in Douglas and Burden.[16] [16]

$$
\Psi(W+\Delta W)=\Psi(W)+\frac{d \Psi}{d W}\{\Delta W\}+O(\Delta W)^{2}
$$

where;

$$
\frac{d \Psi}{d W}=\lambda\left[A_{a}\right]+[K]-\left[K_{T}\right]+[N 1]+[N 2]=\left[K_{\mathrm{tan}}\right]
$$

Thus, the Newton-Raphson iteration procedure for the determination of the post-buckling deflection can be expressed as follows

$\left[K_{\mathrm{tan}}\right]_{i}\{\Delta W\}_{i+1}=-\Psi(W)_{i}$

So, $\{\Delta W\}_{i+1}=-\left[K_{\tan }\right]_{i}^{-1} \Psi(W)_{i}$

where the tangent stiffness matrix $\left[\mathrm{K}_{\mathrm{tan}}\right]_{\mathrm{i}}$ is

$$
\begin{aligned}
& {\left[K_{\text {tan }}\right]_{i}=\lambda\left[A_{a}\right]+[K]-\left[K_{T}\right]+[N 1]_{i}+[N 2]_{i}} \\
& \{W\}_{i+1}=\{W\}_{i}+\{\Delta W\}_{i+1}
\end{aligned}
$$

Convergence occurs in the above procedure when the maximum magnitude of the vector $\{\Delta \mathrm{W}\}_{\mathrm{i}+1}$ becomes less than a given tolerance $\varepsilon_{\mathrm{tol}}$; i.e. $\max \left|\{\Delta \mathrm{W}\}_{\mathrm{i}+1}\right| \leq \varepsilon_{\mathrm{tol}}$.

\section{Free Vibration}

From equation (26), the equation of free vibration about a statically stable position could be stated as

$$
[M]\{\ddot{W}\}_{t}+\left([K]-\left[K_{T}\right]+[N 1]_{s}+[N 2]_{s}\right)\{W\}_{t}=0
$$

or

$$
[M]\{\ddot{W}\}_{t}+\left[K_{\text {tan }}\right]\{W\}_{t}=0
$$

The solution of equation (35) is assumed to take the harmonic form 


$$
\{W\}_{t}=\bar{c}\{\Phi\} e^{\Omega t}
$$

Substituting equation (36) into equation (35) hence, the associated Eigen value problem can be stated as

$$
\left(\Omega^{2}[M]+\left[K_{\tan }\right]\right)\{\Phi\}=0
$$

Thus, the solution procedure would be, first, the solution of the static thermal deflection and the associated stiffness matrices by following the procedure outlined in the preceding section, and then, solving the eigen value problem of equation (37) for the free vibration of a thermally buckled traditional composite material plate.

\section{Panel Flutter under Thermal Effect}

In this section, the procedure of determining the critical non dimensional dynamic pressure under the presence of thermal loading is presented. Equation (26) can be reduced for the solution of the linear (pre-buckling and pre-flutter) problem to the following equation

$$
[M]\{\ddot{W}\}_{t}+[G]\{\dot{W}\}_{t}+\left(\lambda\left[A_{a}\right]+[K]-\left[K_{T}\right]\right)\{W\}_{t}=0
$$

Now apply a new definition for the bending degrees of freedom by considering the transverse deflection degrees of freedom

$$
\left\{W_{B}\right\}=\left\{W_{b}\right\}
$$

Neglecting the in-plane inertia terms will not bring significant error, because their natural frequencies are usually 2 to 3 orders of magnitude higher than the bending ones by Ibrahim [14] and Tawfik [17]. Separating equations (38) in the membrane and transverse directions results in the following transverse dynamic equation

$$
\left[M_{B}\right]\left\{\ddot{W}_{B}\right\}_{t}+\left[G_{B}\right]\left\{\dot{W}_{B}\right\}_{t}+\left(\lambda\left[A_{a B}\right]+\left[K_{B}\right]-\left[K_{T B}\right]\right)\left\{W_{B}\right\}_{t}=0
$$

Now, assume the deflection function of the transverse displacement $\left\{W_{B}\right\}_{t}$ to be in the harmonic form

$$
\left\{W_{B}\right\}_{t}=c\left\{\Phi_{B}\right\} e^{\Omega t}
$$

where $\Omega=\alpha+i \omega$, is the complex panel motion parameter ( $\alpha$ is the damping ratio, and $\omega$ is the frequency), by Xue [18], $c$ is the amplitude of vibration, and $\left\{\Phi_{B}\right\}$ is the mode shape. Substituting equation (40) into (39), the following equation is obtained

$$
c\left[-\kappa\left[M_{B}\right]+\left[\bar{K}_{B}\right]\right]\left\{\Phi_{B}\right\} e^{\Omega t}=0
$$

where $\quad\left[G_{B}\right]=\omega_{o} g_{a}\left[M_{B}\right], \quad \kappa=-\Omega^{2}-\omega_{o} g_{a} \Omega \quad$ and $\quad\left[\bar{K}_{B}\right]=\lambda\left[A_{a B}\right]+\left[K_{B}\right]-\left[K_{T B}\right]$

From equation (41), the generalized Eigen value problem can be written as

$$
\left|-\kappa\left[M_{B}\right]+\left[\bar{K}_{B}\right]\right|=0
$$

Given that the values of $\kappa$ are real for all values of $\lambda$ below the flutter critical value, an iterative solution can be utilized to determine the flutter non-dimensional dynamic pressure $\lambda_{\text {cr }}$ at the given temperature rise. 


\section{Optimization}

Genetic Algorithm is adopted on a finite element code to optimize the shape scheme of laminas of an applied composite material that causes the apparent change in the critical temperature and obtain the best temperature as the maximum one.

Genetic Algorithm (GA) is a distinct optimization code that will be investigated, using of GA has gained some popularity in optimization, and has been identified as a potential technique to be evaluated in heuristic search and combination problems. GA is a global search technique. GA does not require any mathematical increase to the numerical solution methods used to represent the ill objects.

GAs differ from others which is more widely in real valued search space. Search using GAs strikes a reasonable balance between exploiting the available information and searching through unexplored regions.

GA depends on the performance of a population of recommended solution. Population is created from the variable that will be optimized. Genetic Algorithm operation close to the laws of nature, uses a population of potential solutions represented by string of binary digits, called chromosomes or individuals, which is submitted to many transformations, called genetic operations such that selection, crossover and mutation. The population is going to evolve during the generations according to the fitness value of the individuals; then, when a stationary state is reached, the population has converged to the solution of the given optimization problem.

GAs can work as a Multi-Objective Optimization (MOO) where several scores must be satisfied simultaneously to obtain an optimal solution. Extend optimization theory by permitting multiple objectives to be optimized simultaneously. MOO is known as multicriteria, multidisciplinary, or a vector optimization problem. GA is a powerful code because it can be adapted to operate as a parallel genetic algorithm operator.

\section{Working principles}

Wahba [19] mentioned that the basic structure of a GA consists mainly of the following steps:

(1) Random initialization of a population of individuals.

(2) Evaluation of the individuals.

(3) Application of genetic operators (selection, crossover and mutation) to the population and return to step (2) until the best individual is reached.

\section{Parametric study and recommendations}

Experiences clear that crossover rate generally should be high as mentioned by Wahba [19], about $60 \%-80 \%$. On the other hand, the mutation rate should be very low. Best rates reported are about $0.5 \%-1 \%$ (recommended that there is a relationship between mutation rate, population size and chromosome length). It may be surprising, that very big population size usually does not improve performance of GA (in meaning of speed of finding solution), and good population size was found to be around 25-100. In this case sensitivity study is performed and the shape scheme $[0 /-45 / 45 / 90]_{S}$ is optimized to be $[-79 / 46 /-42 / 8]_{S}$ and $[55 /-$ $52 /-77 /-52]_{S}$ for clamped and simply supported flat plate element, respectively. Choose crossover $80 \%$, generation 10 , and population 40 . 


\section{Numerical Results and Discussions}

Numerical analysis of the aero-thermal buckling of a traditional composite plate is performed using the finite element method. The full plate is modeled with a uniform $4 \times 4$ finite element mesh of four-node rectangular plate elements and consists of eight-layered Graphite-Epoxy matrix with a symmetric stacking sequence with old shape scheme of [0/-45/45/90]s. The four edges are all clamped and simply supported respectively. The plate dimensions are chosen to be $0.381 \times 0.305 \times 0.0013 \mathrm{~m}$. The Young's modulus is a function in the temperature rise. The phase transformation is activated at the room temperature $20^{\circ} \mathrm{C}$. The material properties of applied composite lamina, Ibrahim [14] and Hwang and Moon [20] [20], are shown in Table 1

Table 1 Material properties of Graphite-Epoxy composite lamina

\begin{tabular}{|c|c|}
\hline \multicolumn{2}{|c|}{ Graphite-epoxy } \\
\hline Symbol & Value \\
\hline $\mathrm{E} 1$ & $155\left(1-6.35 \times 10^{-4} \Delta \mathrm{T}\right) \mathrm{GPa}$ \\
\hline $\mathrm{E} 2$ & $8.07\left(1-7.69 \times 10^{-4} \Delta \mathrm{T}\right) \mathrm{GPa}$ \\
\hline $\mathrm{G} 12$ & $4.55\left(1-1.09 \times 10^{-3} \Delta \mathrm{T}\right) \mathrm{GPa}$ \\
\hline $\mathrm{P}$ & $1550 \mathrm{Kg} / \mathrm{m}^{3}$ \\
\hline $\mathrm{N}$ & 0.22 \\
\hline$\alpha 1$ & $-0.07 \times 10^{-6}\left(1-0.69 \times 10^{-3} \Delta \mathrm{T}\right) /{ }^{\circ} \mathrm{C}$ \\
\hline$\alpha 2$ & $30.6 \times 10^{-6}\left(1+0.28 \times 10^{-4} \Delta \mathrm{T}\right) /{ }^{\circ} \mathrm{C}$ \\
\hline
\end{tabular}

Figures $(1,2)$ present the optimum arrangement is compared by the old one to show critical temperature rise for clamped and simply supported symmetric composite plate with the maximum non-dimensional thermal deflections respectively. 16 elements are used because of time consuming of genetic algorithms. Curve shows the new position of the critical temperature and observed delaying which improve the aero-thermal buckling stability. Where the angles are [-79/46/-42/8 $]_{\mathrm{S}}$ for clamped plate and [55/-52/-77/-52 $]_{\mathrm{S}}$ for simply supported plate. The validated figure by Ibrahim [14] are shown below the optimum figures $(1,2)$ shows the effect of temperature on the ratio of maximum lateral deflection / thickness but in the validated figure, the absolute temperature is used (Temperature rise " $\Delta \mathrm{T}$ " = absolute temperature - room temperature). Validation is verified for just normal scheme.

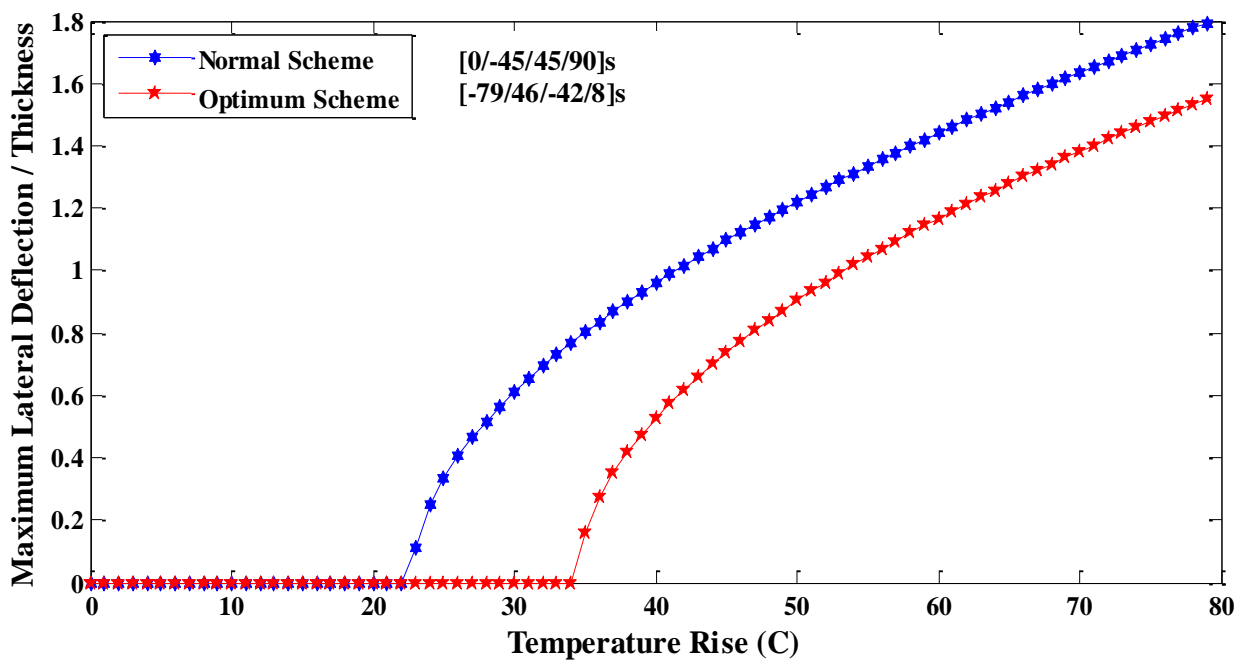

Fig. 1 Optimum and old lamination scheme for symmetric 16 elements clamped composite plate 


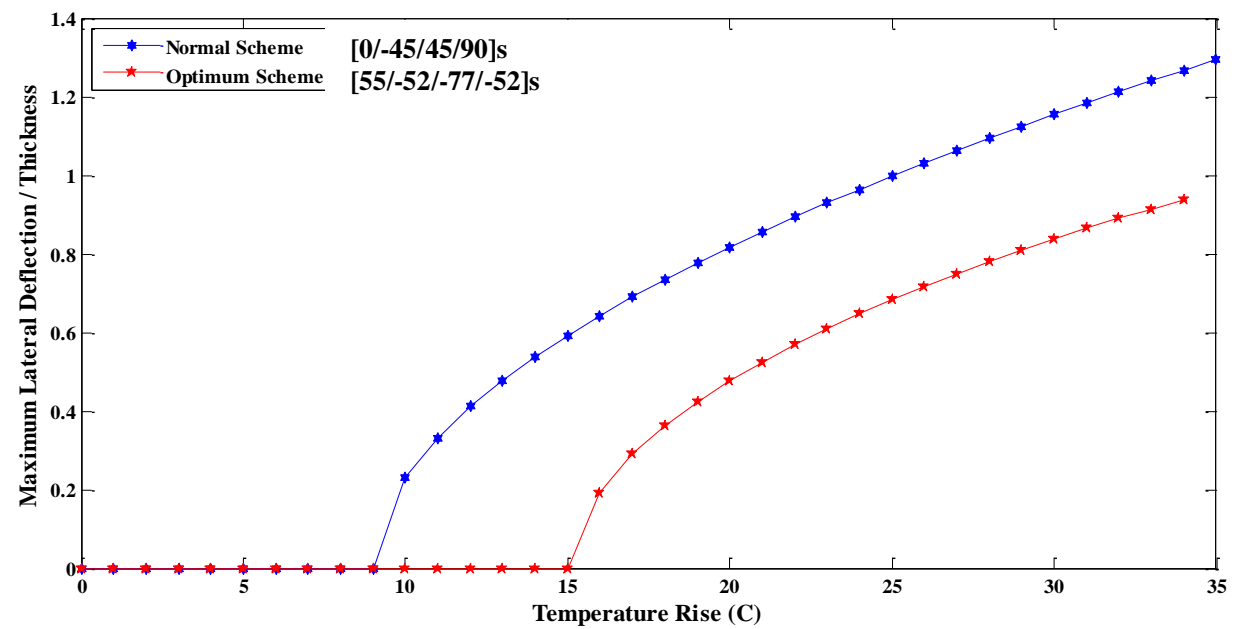

Fig. 2 Optimum and old lamination scheme for symmetric 16 elements simply supported Composite plate

Figures (3) and (4) represent the corresponding Lamination scheme on the fundamental frequency for clamped and simply supported 16 elements traditional composite plate, respectively. These study the effect of temperature rise on the fundamental frequency and demonstrate decreasing in the values of the fundamental frequency while temperature rise increases because of panel lose its stiffness. The critical buckling temperature is found at reflected point of the curve, hence; the nonlinear terms will be added because of theta (angles of fibers) effect is appeared and consequently the fundamental frequency increases again because of stiffness will increases with the temperature rise increasing. Figures (1, 2, 3, and 4) use 16 elements instead of 36 elements because of time consuming of genetic algorithms.

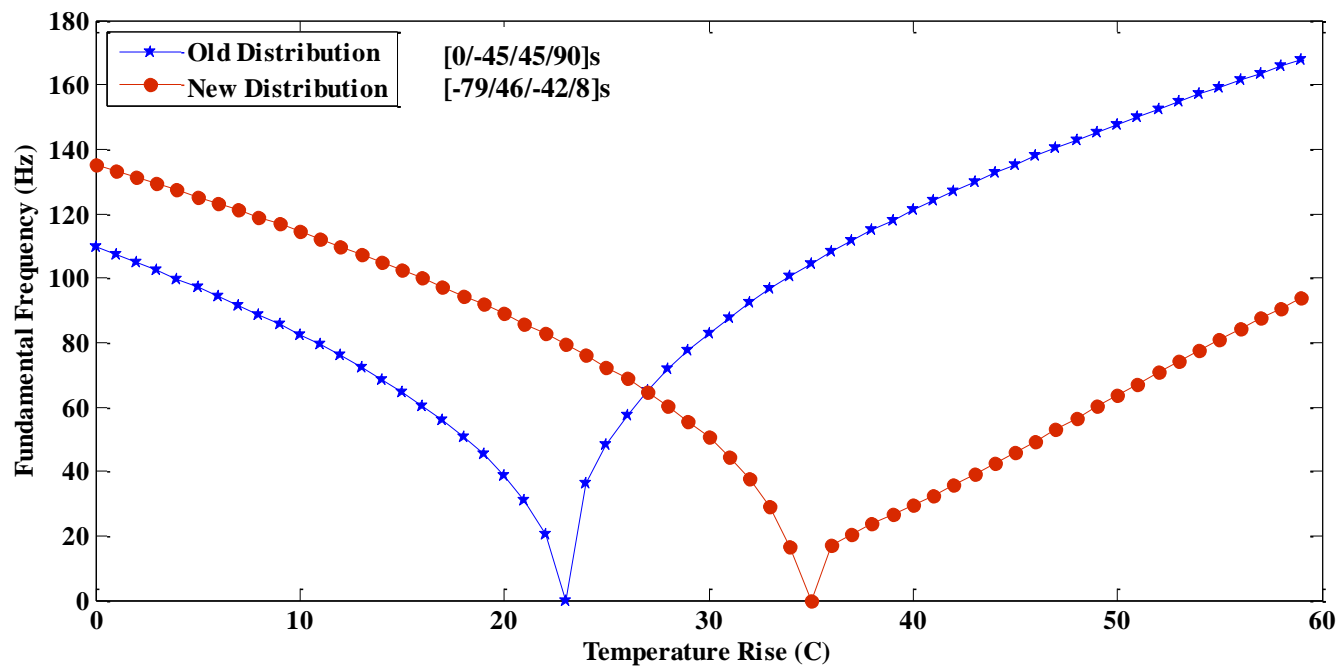

Figure 3 Fundamental frequencies of clamped 16 elements composite plate 


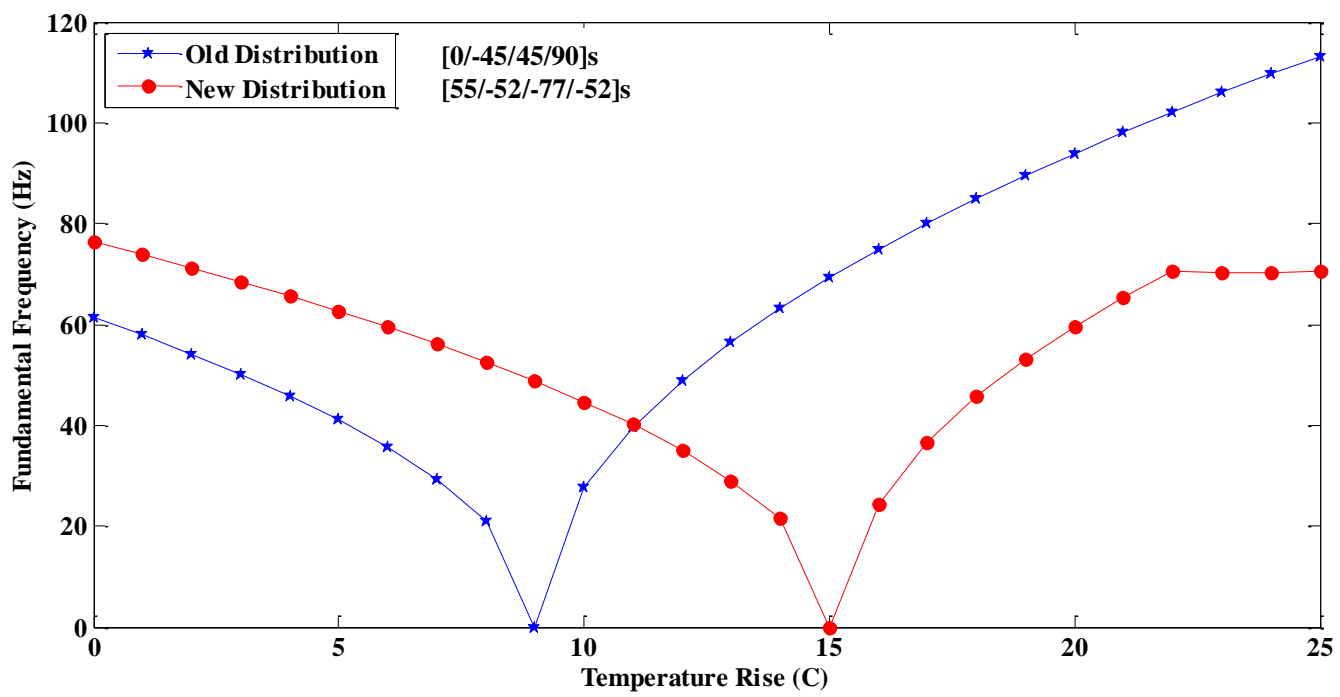

Figure 4 Fundamental frequencies of simply supported 16 elements composite plate

Figure (5) presents a comparison between the stability boundaries of simply supported and clamped 36 elements traditional composite plates. The arrangement of lamination is [0/$45 / 45 / 90]_{\text {s. It }}$ is seen in the figure that the clamped plate has a wider flat and stable region than that of the simply supported one. Figure demonstrates the regions of static and dynamic stability or instability. For two types of fixations, critical non dimensional dynamic pressure decreases while temperature rise increases because of plate lose its stiffness and the corresponding values of dynamic pressure is decreases. So, above this curve; plate is dynamically unstable (panel flutter occurs). Below this curve the plate is flat and stable until appearance of critical buckling temperature curve versus non dimensional dynamic pressure, hence; plate becomes statically unstable. Curves study the phenomena of panel flutter and thermal buckling individually.

\section{Conclusions}

An efficient finite element formulation has been presented for the analysis of supersonic linear panel flutter and thermal buckling characteristics of a composite panel made of graphite epoxy. Conclusions of this work are summarized as follows:

1. Composite panels with simply supported edges were found to have no distinguished buckling phenomena, because any small temperature rise results in a prompt transverse deflection of the panel, due to structural asymmetry about the middle plane.

2. Clamped flat plate panel more stable than simply supported one.

3. Increasing in length over the same thickness decreases the critical temperature (at buckling occurrence).

4. Critical non-dimensional dynamic pressure $\left(\lambda_{\mathrm{cr}}\right)$ decreases with elevated temperature.

5. Improvement and increasing of the critical temperature depend on many parameters as increasing total thickness, material properties, changing thickness of the layers into the same total thickness, and changing orientation scheme. 


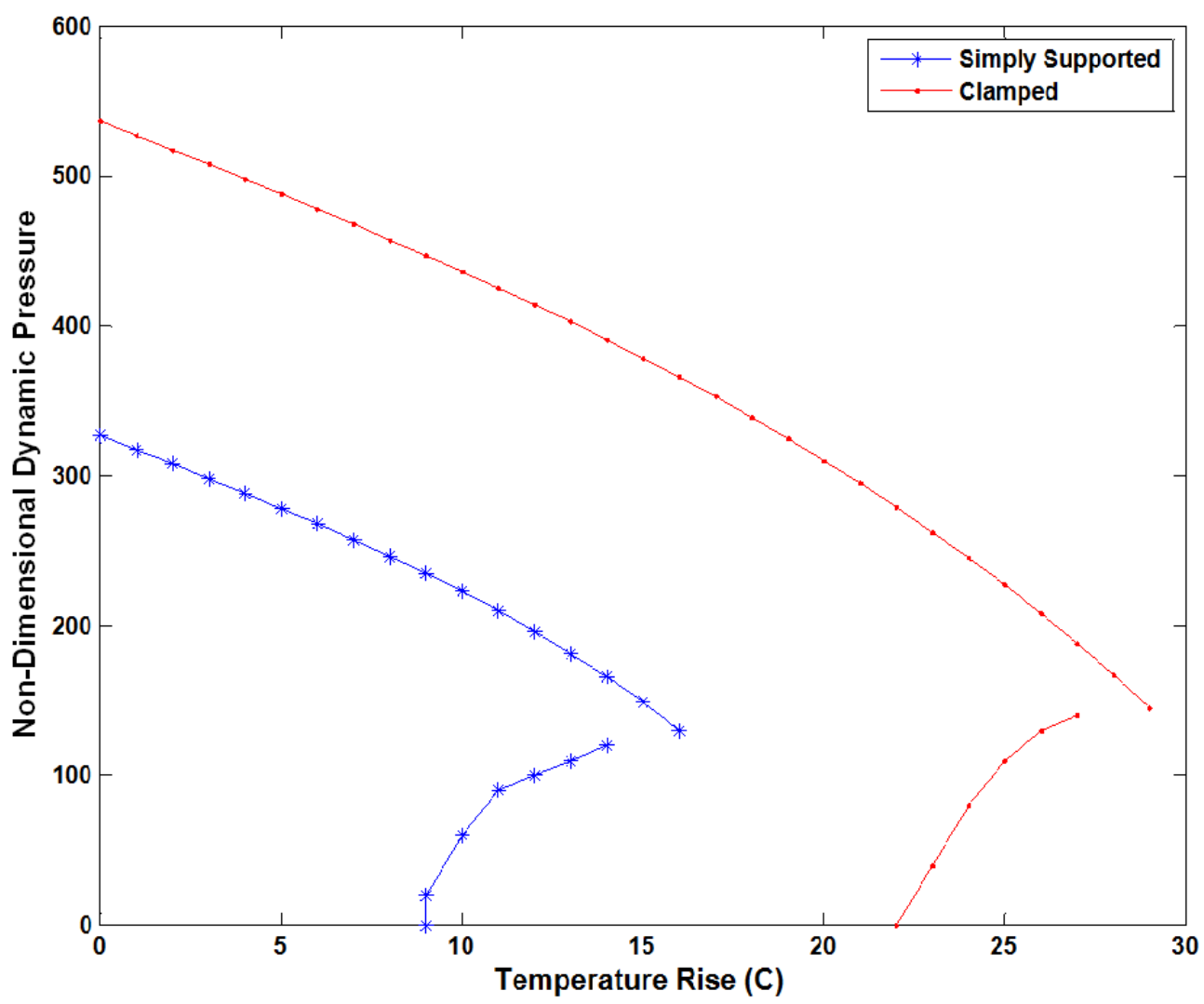

Figure 5 Comparison between the stability boundaries of simply supported and clamped plates

\section{References}

[1] H. H. Ibrahim, M. Tawfik, and H. M. Negm, "Thermal Post-Buckling and Flutter Behavior of Shape Memory Alloy Hybrid Composite Plates", proceedings of the Eighth International Congress of Fluid Dynamics \& Propulsion (ICFDP8), Sharm El-Shiekh, Egypt, American Society of Mechanical Engineers Paper ICFDP-EG-153, Dec. 2006

[2] Young-Shin Lee, Yeol-Wha Lee, Myung-Seog Yang, and Bock-Sun Park, "Optimal Design of Thick Laminated Composite Plates for Maximum Thermal Buckling Load", Journal of Thermal Stresses, Vol. 22, pp. (259-273), 1999

[3] Ji-Ho Kang and Chun-Gon Kim, "Minimum-Weight Design of Compressively Loaded Composite Plates and Stiffened Panels for Post-Buckling Strength by Genetic Algorithm", Journal of Composite Structures, Vol. 69, pp. (239-246), 2005

[4] B. Paluch, M. Grediac, and A. Faye, "Combining A Finite Element Program and A Genetic Algorithm to Optimize Composite Structures with Variable Thickness", Journal of Composite Structures, Vol. 83, pp. (284-294), 2008

[5] Roberto Spallino and Georg Thierauf, "Thermal Buckling Optimization of Composite Laminates by Evolution Strategies", Journal of computers and Structures, Vol. 78, pp. (691 - 697), 2000

[6] A.R. de Faria and S.F.M. de Almeida, "Buckling Optimization of Plates With Variable Thickness Subjected to Non-Uniform Uncertain Loads", International Journal of Solids and Structures, Vol. 40, pp. (3955-3966), 2003

[7] Wu Lanhe, "Thermal Buckling of A Simply Supported Moderately Thick Rectangular FGM Plate", Journal of Composite Structures, Vol. 64, pp. (211-218), 2004 
[8] Somenath Mukherjee, M Manjuprasad, S Deepa Sakravarthini, and R Avinash, "Finite Element Studies on Supersonic Panel Flutter Under High Thermal Environment With Arbitrary Flow Direction", Proceedings of the International Conference on Aerospace Science and Technology, Bangalore, India, 26 - 28 June 2008

[9] H. H. Ibrahim, M. Tawfik, and M. Al-Ajmi, "Non-Linear Panel Flutter For Temperature-Dependent Functionally Graded Material Panels", computational mechanics, Vol. 41, pp. (325-334), 2008

[10] S. TIMOSHENKO and S. WOINOWSKY-KRIEGER, "Theory of Plates And Shells", McGraw-Hill, Inc, 1959

[11] Eduard Ventsel and Theodor Krauthammer, "Thin Plates And Shells", ISBN: 0-82470575-0, Publisher Marcel Dekker, 2001

[12] NASA Reference Publication 1351, "Basic Mechanics of Laminated Composite Plates", A. T. Nettles, October 1994

[13] Reddy, J. N., "Theory And Analysis of Elastic Plates", Taylor \&Francis, 1999

[14] H. H. Ibrahim, "The Effect of Shape Memory Alloy Embeddings on The Nonlinear Flutter of Composite Panels Subject to Thermal and Random Acoustic Loads", PHD Thesis, Faculty of Engineering, Cairo University, GIZA, EGYPT, 2007

[15] Dowell, E. H., "Aero-Elastic of Plates And Shells", Noordhoff International Publishing, the Netherlands, 1975

[16] J. Douglas Faires and Richard L. Burden, "Numerical Methods", third edition, Brooks Cole, june 18, 2002

[17] Tawfik, M., "Suppression of Post-Buckling Deflection and Panel Flutter Using Shape Memory Alloy", M.Sc Thesis, Old Dominion University, Norfolk, VA, December 1999

[18] Xue, D. Y., "Finite Element Frequency Domain Solution of Nonlinear Panel Flutter with Temperature Effects And Fatigue Life Analysis", PhD dissertation, Old Dominion University, Mechanical Engineering Department, Norfolk, Virginia, 1991

[19] Waleed A.M. Wahba, "Design Optimisation of Centrifugal Pump Impellers Using Parallel Genetic Algorithm", Ph.D. Thesis, Cranfield University, September 2001

[20] Seong Hwan Moon and Joon Seok Hwang, "Panel Flutter Suppression with an Optimal Controller Based on The Nonlinear Model Using Piezoelectric Materials", journal of Composite Structures, Vol. 68, pp. (371-379), 2005 